\title{
Decomposition of Minkowski-Rådström-Hörmander Space to the Direct Sum of Symmetric and Asymmetric Subspaces
}

\author{
Jerzy Grzybowski • Hubert Przybycień • \\ Ryszard Urbański
}

Received: 26 May 2012 / Accepted: 25 January 2013 /

Published online: 6 March 2013

(C) The Author(s) 2013. This article is published with open access at Springerlink.com

\begin{abstract}
Any abstract convex cone $S$ with a uniformity satisfying the law of cancellation can be embedded in a topological vector space $\widetilde{S}$ (Urbański, Bull Acad Pol Sci, Sér Sci Math Astron Phys 24:709-715, 1976). We introduce a notion of a cone symmetry and decompose in Theorem 2.12 a quotient vector space $\widetilde{S}$ into a topological direct sum of its symmetric subspace $\widetilde{S}_{s}$ and asymmetric subspace $\widetilde{S}_{a}$. In Theorem 2.19 we prove a similar decomposition for a normed space $\widetilde{S}$. In section 3 we apply decomposition to Minkowski-Rådström-Hörmander (MRH) space with three best known norms and four symmetries. In section 4 we obtain a continuous selection from a MRH space over $\mathbb{R}^{2}$ to the family of pairs of nonempty compact convex subsets of $\mathbb{R}^{2}$.
\end{abstract}

Keywords Quasidifferential - Abstract convex cone - Cone of nonempty bounded closed convex sets • Minkowski-Rådström-Hörmander space • Hausdorff metric • Demyanov metric $\cdot$ Bartels-Pallaschke norm

Mathematics Subject Classifications (2010) $52 \mathrm{~A} 07 \cdot 22 \mathrm{~A} 20 \cdot 46 \mathrm{~N} 10 \cdot 26 \mathrm{~A} 27$

\section{Introduction}

A Minkowski-Rådström-Hörmander space plays important role in quasidifferential calculus. A quasidifferential is a pair of convex sets or, more correctly, a quotient

\footnotetext{
J. Grzybowski $(\bowtie) \cdot$ H. Przybycień · R. Urbański

Faculty of Mathematics and Computer Science, Adam Mickiewicz University,

Umultowska 87, 61-614 Poznań, Poland

e-mail: jgrz@amu.edu.pl

H. Przybycień

e-mail: hubert@amu.edu.pl

R. Urbański

e-mail: rich@amu.edu.pl
} 
class of pairs of convex sets, which is an element of a MRH space. Also a differential of a one-variable multifunction with convex values is an element of a MRH space.

A MRH space $\tilde{X}=\mathcal{B}^{2}(X) / \sim$ is the smallest vector space in which the cone $\mathcal{B}(X)$ of all nonempty bounded closed convex subsets of real Hausdorff topological vector space $X$ can be embedded (see $[4,11,19,20,25]$ ). The relation of MRH space to $\mathcal{B}(X)$ can be compared to the relation of the ring $\mathbb{Z}$ of integers to the semigroup of natural numbers $\mathbb{N}$. A MRH space appears very useful in a theory of generalized differentiation developed by many authors (see for example Rockafellar and Wets [22] and Mordukhovich [14]). A MRH space is closely related to quasidifferential calculus of Demyanov and Rubinov [1,2]. In particular the structure of a vector space enables differentiation of multifunctions (see for example [6]). As MRH spaces are so natural and useful they deserve more study.

In $\mathcal{B}\left(\mathbb{R}^{n}\right)$ Ewald and Shephard [5] introduced the following equivalence relation $A \approx B$ if the set $A+(-B)$ is centrally symmetric. The quotient set $\mathcal{A}^{n}$ of all asymmetry classes $[A]$ with addition $[A]+[B]=[A+B]$, scalar multiplication $\lambda[A]=[\lambda A]$ is a vector space equipped with a norm

$$
\|A\|=\inf _{p \in \mathbb{R}^{n}} d_{H}(A-p, p-A) .
$$

Ratschek and Schröder [21] showed in Lemma that $\mathcal{B}(X)$ (and even any convex cone with multiplication by all real numbers and law of cancellation) is subdirect product of $\mathcal{B}(X) / \approx$ (where $A \approx B$ if $A+(-B)=B+(-A)$ ) and the cone $\mathcal{B}_{S}(X)$ of all symmetric sets.

Markov [13] embedded $\mathcal{B}(X)$ in $\tilde{X}$ where $\lambda *[A, B]=[\lambda A, \lambda B]$ and $(\tilde{X},+, *)$ is so called quasivector space (see Definition 2 in [13]). Besides he decomposed quasivector space $\widetilde{X}$ into direct product of a vector space (our $\widetilde{S}_{a}$, where $S=\mathcal{B}(X)$, with symmetry $T_{0}$; see Definitons 2.2 and 4.3 ) and quasivector space (our $\widetilde{S}_{S}$ with scalar multiplication “*”).

We introduce in Section 2 a notion of a cone symmetry (compare with involution in [13]) for an abstract convex cone $S$ instead of just $\mathcal{B}(X)$. We also introduce symmetric subspace and asymmetric subspace of the quotient space $\widetilde{S}$. Having defined a cone symetry $T$ we decompose in Theorem 2.12 a topological vector space $\underset{\widetilde{S}}{\widetilde{S}}$ into a topological direct sum of its symmetric subspace $\widetilde{S}_{s}$ and asymmetric subspace $\widetilde{S}_{a}$. In Theorem 2.19 we decompose a normed space $\widetilde{S}$. In Section 3 we give examples of decomposition of a Minkowski-Rådström-Hörmander space $\widetilde{X}$ over a normed vector space $X$ where $\tilde{X}$ is equiped with three different but natural norms and cone $\mathcal{B}(X)$ is equiped with four different cone symmetries $T$. Finally, in Section 4, using our decomposition, we define a continuous function (selection) $\Sigma$ from $\left(\widetilde{\mathbb{R}^{2}},\|\cdot\|_{B P}\right)$ to $\left(\mathcal{B}^{2}\left(\mathbb{R}^{2}\right), d_{B P} \times d_{B P}\right)$ such that $\Sigma(\widetilde{x}) \in \tilde{x}$ for all $\widetilde{x} \in \widetilde{\mathbb{R}^{2}}$. Such selection of $\left(\widetilde{\mathbb{R}^{n}},\|\cdot\|\right)$ is impossible for two other norms and probably impossible for $n \geq 3$.

\section{Decomposition of Quotient Group}

In this section first we recall (Proposition 2.4 and Theorem 2.6) main results from [10] concerning commutative semigroup $S$ with 0 satisfying the law of cancellation and a semigroup symmetry $T$ on $S$. 
Definition 2.1 We say that $S$ is 2-torsion-free if $s+s=t+t$ implies $s=t$. We say that a semigroup symmetry $T$ is 2-divisible if for any $s \in S$ there exists $t \in S$ such that $s+T s=t+t$.

Let $\mathcal{U}$ be a uniformity on a commutative semigroup $S$ with the neutral element 0 .

Definition 2.2 The addition + is uniformly continuous if for any $U \in \mathcal{U}$ there exists $V \in \mathcal{U}$ such that $V+V \subset U$, where $V+V=\left\{\left(s+s^{\prime}, t+t^{\prime}\right) \mid(s, t),\left(s^{\prime}, t^{\prime}\right) \in V\right\}$. The addition + is strongly uniformly continuous if for any $U \in \mathcal{U}$ there exists $V \in \mathcal{U}$ such that $V+\left(S^{2} \backslash U\right) \subset S^{2} \backslash V$, where $S^{2}=S \times S$.

Remark 2.3 The strong uniform continuity implies the law of cancellation.

Proposition 2.4 (See Proposition 3.5 in [10]) If the addition in $S$ is strongly uniform continuous then the addition in the quotient group $S^{2} / \sim\left((s, t) \sim\left(s^{\prime}, t^{\prime}\right)\right.$ if and only if $\left.s+t^{\prime}=t+s^{\prime}\right)$ is continuous and the topology in $S^{2} / \sim$ determined by the family $\{\widetilde{\mathcal{U}}=$ $\left.\left\{[(s, t)]_{\sim} \mid(s, t) \in U\right\} \mid U \in \mathcal{U}\right\}$ as the basis of neighborhoods of $[(0,0)]_{\sim}$ is Hausdorff.

Definition 2.5 We say that a topological group $G$ is the direct sum of its subgroups $G_{1}$ and $G_{2}$ if the canonical mapping from $G=G_{1}+G_{2}$ to $G_{1} \times G_{2}$ is a homeomorphism. We say that the symmetry $T$ in $S$ is uniformly continuous if $T(U)=$ $\{(T s, T t) \mid(s, t) \in U\} \in \mathcal{U}$ for all $U \in \mathcal{U}$.

Notice that $T^{-1}(U)=T(U)$.

Theorem 2.6 (See Theorem 3.7 in [10]) Let $S$ be a commutative semigroup with 0 satisfying the law of cancellation and $T$ be a symmetry in $S$. If

(i) the addition + is strongly uniformly continuous,

(ii) the semigroup $S$ is 2-torsion-free,

(iii) for any $U \in \mathcal{U}$ there exists $V \in \mathcal{U}$ such that if $s+s \in V$ and $T s=s$ then $s \in U$,

(iv) the mapping $T$ is uniformly continuous,

(v) $T$ is 2-divisible

then the topological quotient group $S^{2} / \sim$ is the topological direct sum of subgroups $\left\{[(s, t)]_{\sim} \mid T s=s, T t=t\right\}$ and $\left\{[(s+u, t+v)]_{\sim} \mid u+u=T t+t, v+v=T s+s\right\}$.

\subsection{Topological Quotient Spaces}

Now we apply the results on semigroups in order to obtain new results (Theorems 2.10-2.13) on abstract convex cones with uniformity. 
Definition 2.7 We say that a commutative semigroup $S$ with zero is an abstract convex cone [25] if there is also given a mapping $\mathbb{R}_{+} \times S \ni(\lambda, s) \longmapsto \lambda s \in S$ such that

$$
\begin{aligned}
1 s & =s, \\
\lambda(\mu s) & =(\lambda \mu) s, \\
\lambda(s+t) & =\lambda s+\lambda t, \\
(\lambda+\mu) s & =\lambda s+\mu s
\end{aligned}
$$

for all $s, t \in S$ and $\lambda, \mu \in \mathbb{R}_{+}$. In this paper we consider only abstract convex cones satisfying law of cancellation.

The element $a \in S$ is a summand of $b \in S$ if $a+c=b$ for some $c \in S$. By $S_{0}$ we denote the vector space of all summands of 0 which is also called a subspace of linearity.

For $(s, t),\left(s^{\prime}, t^{\prime}\right) \in S^{2}$ let $(s, t) \sim\left(s^{\prime}, t^{\prime}\right)$ if and only if $s+t^{\prime}=s^{\prime}+t$. Denote $\widetilde{S}=$ $S^{2} / \sim$. By $[s, t]$ we denote the quotient class of $(s, t)$. Let $[s, t]+\left[s^{\prime}, t^{\prime}\right]=\left[s+s^{\prime}, t+t^{\prime}\right]$. The opposite element to $[s, t]$ is $[t, s]$. It is well known that if we define the scalar multiplication by $\lambda[s, t]=[\lambda s, \lambda t]$ for $\lambda \geq 0$ and $\lambda[s, t]=[-\lambda t,-\lambda s]$ for $\lambda \leq 0$ then the quotient set $\widetilde{S}$ is a real vector space.

Definition 2.8 We say that $T: S \longrightarrow S$ is a cone symmetry if $T$ is an abstract cone homomorphism, $T^{2} s=s$ for $s \in S$ and $T s=-s$ for $s \in S_{0}$. The element $s \in S$ is called symmetric if $T s=s$. By $S_{s}$ we denote the subsemigroup of $S$ of all symmetric elements. A vector (quotient class) $\tilde{x}_{s}=[s, t]_{s}=\left[\frac{1}{2}(s+T s), \frac{1}{2}(t+T t)\right]$ is called the symmetric part of $\tilde{x}=[s, t] \in \widetilde{S}$ and $\tilde{x}_{a}=[s, t]_{a}=\left[\frac{1}{2}(s+T t), \frac{1}{2}(t+T s)\right]=\tilde{x}-\tilde{x}_{s}$ is called the asymmetric part of $\tilde{x}$. A subspace $\widetilde{S}_{s}=\{[s, t] \mid s, t \in S, T s=s, T t=t\}$ is called the symmetric subspace of $\widetilde{S}$ and $\widetilde{S}_{a}=\{[s+T t, t+T s] \mid s, t \in S\}$ is called the asymmetric subspace of $\widetilde{S}$.

The mappings $\operatorname{pr}_{s}: \widetilde{S} \longrightarrow \widetilde{S}_{S}, \operatorname{pr}_{s}(\tilde{x})=\tilde{x}_{s}$ and $\operatorname{pr}_{a}: \widetilde{S} \longrightarrow \widetilde{S}_{a}, \operatorname{pr}_{a}(\tilde{x})=\tilde{x}_{a}$ are linear projections.

Definitions 2.7 and 2.8 and the notations were used in [10] in the case of commutative, cancellative semigroups with 0 .

Definition 2.9 We say that a vector space $V$ is the direct sum of its subspaces $V_{1}, V_{2}$ and write $V=V_{1} \oplus V_{2}$ if $V=V_{1}+V_{2}$ and $V_{1} \cap V_{2}=\{0\}$.

The following theorem is a corollary from Theorem 2.7 in [10], which was proved for commutative semigroups.

Theorem 2.10 Let $S$ be an abstract convex cone and $T: S \longrightarrow S$ a cone symmetry then $\widetilde{S}=\widetilde{S}_{a} \oplus \widetilde{S}_{s}$ where $\widetilde{S}_{a}$ and $\widetilde{S}_{s}$ are linear subspaces of $\dot{\widetilde{S}}$. 
The proof is easy and we omit it. For a cone $S$ which is also a uniform space the following theorem holds true:

Theorem 2.11 If a uniformity $\mathcal{U}$ on abstract cone $S$ satisfies the following conditions:

(i) the addition + is strongly uniformly continuous,

(ii) for every $U \in \mathcal{U}$ we have $\bigcup_{\lambda>0} \lambda U=S \times S$,

(iii) for every $U \in \mathcal{U}$ there exists $V \in \mathcal{U}$ and $\lambda>0$ such that $[0, \lambda] V \subset U$

then the topology in $\widetilde{S}$ determined by the family $\{\widetilde{\mathcal{U}}=\{[s, t] \mid(s, t) \in U\} \mid U \in \mathcal{U}\}$ as the basis of neighborhoods of $\tilde{0}$ is Hausdorff.

Theorem 2.11 follows from Proposition 2.4. Analogous uniformities were considered in [25]. Now, from Theorem 2.11 we obtain the following central theorem on the decomposition of a quotient topological vector space $\widetilde{S}$ :

Theorem 2.12 Let $S$ be an abstract convex cone and let $\mathcal{U}$ be a uniformity on $S$ satisfying the following conditions:

(i) the addition + is strongly uniformly continuous,

(ii) for every $U \in \mathcal{U}$ we have $\bigcup_{\lambda>0} \lambda U=S \times S$,

(iii) for every $U \in \mathcal{U}$ there exists $V \in \mathcal{U}$ and $\lambda>0$ such that $[0, \lambda] V \subset U$.

If $T$ is a uniformly continuous cone symmetry then the topological vector space $\widetilde{S}$ is the topological direct sum of its subspaces $\widetilde{S}_{a}$ and $\widetilde{S}_{s}$.

Let $\kappa: S \longrightarrow \widetilde{S}$ be a canonical embedding $\kappa(t)=[t, 0]$. The following theorem is a corollary from Theorem 2.12.

Theorem 2.13 Let $S$ be an abstract convex cone, $V$ be subspace of $S_{0}$ and let $\mathcal{U}$ be a uniformity on $S$ satisfying assumptions of Theorem 2.11. If the mapping $S_{0} \ni t \mapsto$ $-t \in S_{0}$ is uniformly continuous then the following conditions are equivalent:

(a) the space $\widetilde{S}$ has a subspace complementary to the subspace $\kappa(V)$,

(b) there exists a uniformly continuous homomorphic mapping $\sigma: S \longrightarrow V$, such that $\sigma(\sigma(t))=\sigma(t)$ for $t \in S$ and $\sigma(S)=V$.

Proof Suppose $\widetilde{S}=\kappa(V) \oplus W$ is a topological direct sum. Then the canonical projection $\pi=\pi_{\kappa(V)}: \widetilde{S} \longrightarrow \kappa(V)$ is uniformly continuous linear function. Let $\sigma$ be a superposition $\kappa^{-1} \circ \pi \circ \kappa$. Then $\sigma$ is a uniformly continuous cone-homomorphic function such that $\sigma \circ \sigma=\sigma$ and $\sigma(S)=V$.

Now, suppose $\sigma$ satisfies condition (b) of our theorem. Define mapping $T: S \longrightarrow$ $S$ by $T s=s+(-2 \sigma(s)), s \in S$. It is easy to observe that $T$ is a uniformly continuous cone symmetry and $\widetilde{S}_{a}=\kappa(V)$. By Theorem 2.12 we have $\widetilde{S}=\kappa(V) \oplus \widetilde{S}_{s}$. 


\subsection{Normed Quotient Spaces}

Now we apply the results from the previous subsection to abstract convex cones equipped with metric compatible with addition and multiplication by nonnegative scalars.

Definition 2.14 Let $S$ be an abstract convex cone with zero. We say that metric $d$ : $S \times S \longrightarrow \mathbb{R}_{+}$is compatible with addition and scalar multiplication if $d(s, t)=d(s+$ $u, t+u)$ and $d(\lambda s, \lambda t)=\lambda d(s, t)$ for all $s, t, u \in S, \lambda \in \mathbb{R}_{+}$.

The following proposition holds true.

Proposition 2.15 Let $S$ be an abstract convex cone with zero. Let $d: S \times S \longrightarrow \mathbb{R}_{+}$ and $\|\cdot\|: \widetilde{S} \longrightarrow \mathbb{R}_{+}$be such functions that $\|[s, t]\|=d(s, t)$. Then the function $d$ is $a$ metric compatible with addition and scalar multiplication if and only if the function $\|\cdot\|$ is a norm in $\widetilde{S}$.

Definition 2.16 Let $S$ be an abstract convex cone and $d$ be a metric on $S$ compatible with the addition and scalar multiplication on $\mathrm{S}$. We say that the uniformity $\mathcal{U}$ on $S$ given by the basis $\left\{U_{\varepsilon}\right\}_{\varepsilon>0}$, where $U_{\varepsilon}=\{(s, t) \mid d(s, t)<\varepsilon\}$ is the uniformity generated by metric $d$.

It is easy to observe that the following propositions and theorem hold true.

Proposition 2.17 Let $S$ be an abstract convex cone and d be a metric on $S$ compatible with the addition and scalar multiplication on $S$. Then the uniformity $\mathcal{U}$ generated by metric d fulfills the assumptions (i)-(iii) of Theorem 2.11. Moreover the topology in $\tilde{S}$ generated by the uniformity $\mathcal{U}$ is identical with the topology generated by the norm $\|[s, t]\|=d(s, t)$.

Proposition 2.18 Let $S$ be an abstract convex cone and d be a metric on $S$ compatible with the addition and scalar multiplication on $S$. A cone symmetry $T: S \rightarrow S$ is uniformly continuous with respect to the metric $d$ if and only if it is uniformly continuous on $(S, \mathcal{U})$ where $\mathcal{U}$ is the uniformity generated by $d$.

Theorem 2.19 Let $S$ be an abstract convex cone and $d$ be a metric on $S$ compatible with the addition and scalar multiplication on $S$ and let $T: S \rightarrow S$ be a uniformly continuous cone symmetry. Then the normed space $(\widetilde{S},\|\cdot\|)$ where $d(s, t)=\|[s, t]\|$ is a topological direct sum of its subspaces $\widetilde{S}_{s}$ and $\widetilde{S}_{a}$.

\section{Decomposition of a MRH Space}

Let $X$ be a Hausdorff topological vector space and $\mathcal{B}(X)$ the family of all nonempty closed convex bounded subsets of $X$. For $A, B \in \mathcal{B}(X), \lambda \in \mathbb{R}$ we define $A \dot{+} B=$ $\operatorname{cl}\{a+b \mid a \in A, b \in B\}$ and $\lambda A=\{\lambda a \mid a \in A\}$. Then $\mathcal{B}(X)$ is an abstract convex cone with zero and $\tilde{X}=\mathcal{B}^{2}(X) / \sim$ is a Hausdorff topological vector space (well known Minkowski-Rådström-Hörmander space [25]). 
Theorem 2.19 enables us to decompose a MRH space $\tilde{X}$ into topological direct sum of its subspaces generated by a cone symmetry $T$ as long as $T$ is uniformly continuous with respect to given metric $d$. Markov [13] decomposed a MRH space only algebraicly and only in the case of $T A=-A$ (our symmetry $T_{0}$ from Definition 3.3). We will give examples of three more cone symmetries and decompose a MRH space topologicaly with respect to three known metrics.

Let $X$ be a normed space. Let us remind three well known metrics in the cone of convex sets.

Definition 3.1 Let $A, B \in \mathcal{B}(X)$.

1. Hausdorff metric is defined by

$$
d_{H}(A, B)=\inf \{\varepsilon \geq 0 \mid A \subset B+\varepsilon \mathbb{B}, B \subset A+\varepsilon \mathbb{B}\},
$$

where $\mathbb{B}$ is the closed unit ball in $X$.

2. Bartels-Pallaschke metric is defined by

$$
d_{B P}(A, B)=\inf \{\|C\|+\|D\| \mid(C, D) \in[A, B]\},
$$

where $\|A\|=\sup \{\|x\| \mid x \in A\}$.

3. Demyanov's generalized metric (see Grzybowski and Przybycień (submitted)) is defined by

$$
d_{D}(A, B)=\sup \left\{\frac{\left|\left(p_{A}-p_{B}\right)\left(y_{1}\right)-\left(p_{A}-p_{B}\right)\left(y_{2}\right)\right|}{\left\|y_{1}-y_{2}\right\|} \mid y_{1}, y_{2} \in X^{\star}, y_{1} \neq y_{2}\right\},
$$

where $p_{A}(y)=\sup \{y(x) \mid x \in A\}$.

Notice that $p_{A}$ is the support function of a set of A and $X^{\star}$ is the topological dual of $X$ with its usual norm. For $X=\mathbb{R}^{n}$ the metric $d_{D}$ is identical with Demyanov's metric (also called Pliś' metric; see [2] and [12]). All three metrics are complete in the cone $\mathcal{B}\left(\mathbb{R}^{n}\right)$. More information on these three metrics can be found in [3] and Grzybowski and Przybycień (submitted).

Proposition 3.2 The following inequalities hold true:

$$
d_{H} \leq d_{D} \leq d_{B P}
$$

Proof Consider two sets $A, B \in \mathcal{B}(X)$. Let us assume that for any positive $c<$ $d_{H}(A, B)$ the sum $B+c \mathbb{B}$ does not contain the set $A$. By $\mathbb{B}$ we denote the unit ball in the normed space $X$. Let us fix $c$ and choose any element $a$ of $A \backslash(B+c \mathbb{B})$. By the separation theorem there exists a continuous linear functional $f$ with norm 1 such that

$$
p_{A}(f) \geq f(a) \geq \sup _{x \in B+c \mathbb{B}} f(x)=\sup _{b \in B} f(b)+c=p_{B}(f)+c .
$$

Hence, putting $y_{1}=f$ and $y_{2}=0$, we obtain

$$
d_{D}(A, B) \geq \frac{\left|\left(p_{A}-p_{B}\right)(f)-\left(p_{A}-p_{B}\right)(0)\right|}{\|f-0\|} \geq c .
$$


On the other hand, let $y_{1}, y_{2} \in X^{\star}$ and $p_{A}\left(y_{1}\right)=y_{1}\left(a_{1}\right)+\varepsilon_{1}, p_{A}\left(y_{2}\right)=y_{2}\left(a_{2}\right)+$ $\varepsilon_{2}$ for some nonnegative numbers $\varepsilon_{1}, \varepsilon_{2}$ and elements $a_{1}, a_{2} \in A$. Then $y_{2}\left(a_{2}\right)+\varepsilon_{2} \geq$ $y_{2}\left(a_{1}\right)$, and

$$
\begin{aligned}
p_{A}\left(y_{1}\right)-p_{A}\left(y_{2}\right) & =y_{1}\left(a_{1}\right)+\varepsilon_{1}-y_{2}\left(a_{2}\right)-\varepsilon_{2} \\
& \leq y_{1}\left(a_{1}\right)+\varepsilon_{1}-y_{2}\left(a_{1}\right) \leq\left\|y_{1}-y_{2}\right\| \cdot\|A\|+\varepsilon_{1},
\end{aligned}
$$

where $\|A\|=\sup _{a \in A}\|a\|$. Since $\varepsilon_{1}$ can be arbitrarily small, we obtain $\mid p_{A}\left(y_{1}\right)-$ $p_{A}\left(y_{2}\right) \mid \leq\left\|y_{1}-y_{2}\right\| \cdot\|A\|$. Hence

$$
\frac{\left|\left(p_{A}-p_{B}\right)\left(y_{1}\right)-\left(p_{A}-p_{B}\right)\left(y_{2}\right)\right|}{\left\|y_{1}-y_{2}\right\|} \leq\|A\|+\|B\| .
$$

Therefore, $d_{D}(A, B) \leq\|A\|+\|B\|$. Let us observe, that if $(C, D) \in[A, B]$ then $p_{C}-p_{D}=p_{A}-p_{B}$. It implies that $d_{D}(A, B) \leq d_{B P}(A, B)$.

Proposition 3.2 generalizes known results for finite dimesional space. Respective metrics turn MRH spaces $\tilde{X}$ into normed spaces with norms $\|\cdot\|_{H},\|\cdot\|_{D}$ and $\|\cdot\|_{B P}$, respectively. However, only the last norm turns the space $\widetilde{X}$ into a Banach space (compare Theorem 3.4.5 in [16]).

Let us define four cone symmetries.

Definition 3.3 Let $A \in \mathcal{B}(X)$.

(1) Let $T_{0}$ be a central symmetry with the center in 0: $T_{0} A=-A=\{-a \mid a \in A\}$.

(2) Let $X=\mathbb{R}^{n}$ and $T_{S} A=A-2 s A$, where $s A$ is the Steiner's point of $A$ [23].

(3) Let $X=\mathbb{R}^{n}$ and $T_{M} A=A-2 m A$, where $m A$ is the maximal element of $A$ with respect to lexicographical ordering, i.e. $y$ follows $x$ if and only if $x_{i}<y_{i}$ for some $i$ and $x_{j}=y_{j}$ for all $j<i$.

(4) Let $R: X \longrightarrow X$ be a continuous linear mapping such that $R^{2}(A)=A$ for all $A \in \mathcal{B}(X), A=-A$, e.g. $X=\mathbb{R}^{2}$ and $R$ is a rotation around 0 by right angle. We denote $T_{R} A=R(A)$.

We chose these four symmetries in order to illustrate Theorem 2.19. The symmetry $T_{0}$ is probably the most natural. The symmetries $T_{S}$ and $T_{M}$ are similar. They depend on continuous selection of points from convex sets. The symmetry $T_{M}$ can be extended to compact convex sets in separable normed space. Both symmetries give a continuous projection of $\widetilde{X}$ on $X$.

Proposition 3.4 A cone symmetry $T$ is continuous with respect to metric $d$ for all $(T, d) \in\left\{T_{0}, T_{S}, T_{M}, T_{R}\right\} \times\left\{d_{H}, d_{D}, d_{B P}\right\}$ with the exception of $\left(T_{M}, d_{H}\right)$. Moreover, the symmetry $T$ is a Lipschitzian function, i.e. for all convex sets $A, B$ we have $d(T A, T B) \leq \lambda d(A, B)$, where the constant $\lambda=\lambda(T, d)$ is listed in the following table:

\begin{tabular}{|c|c|c|c|c|}
\hline$\lambda$ & $T_{0}$ & $T_{S}$ & $T_{M}$ & $T_{R}$ \\
\hline$d_{H}$ & 1 & $1+2 \kappa_{n}\left\|\mathbb{B}^{e}\right\|$ & $\infty$ & $\|R(\mathbb{B})\|$ \\
\hline$d_{D}$ & 1 & $1+2 \kappa_{n}\left\|\mathbb{B}^{e}\right\|$ & 3 & $\|R(\mathbb{B})\|$ \\
\hline$d_{B P}$ & 1 & $1+2 \kappa_{n}\left\|\mathbb{B}^{e}\right\|$ & 3 & $\|R(\mathbb{B})\|$ \\
\hline
\end{tabular}


The constant $\kappa_{n}$ is given in [18]. The number $\|A\|$ is the smallest radius of a closed ball containing $A$. The unit ball is denoted by $\mathbb{B}$ and Euclidean unit ball is denoted by $\mathbb{B}^{e}$.

Proof Since symmetry $T_{0}$ is an isometric mapping in considered metric spaces, the constant $\lambda=\lambda\left(T_{0}, d\right)=1$ for all three metrics.

We know that $\|s A-s B\|_{2} \leq \kappa_{n} d_{H, 2}(A, B)$ (see [18], for example $\kappa_{2}=\frac{4}{\pi}$ ) for Euclidean norm $\|\cdot\|_{2}$ in $n$-dimensional vector space and Hausdorff metric $d_{H, 2}$ induced by the same norm. Also the following inequalities hold true for respective norms and metrics: $\|\cdot\| \leq\left\|\mathbb{B}^{e}\right\|\|\cdot\|_{2}$ and $d_{H, 2} \leq\left\|\mathbb{B}^{e}\right\| d_{H}$. Then, by translational invariance of considered metrics, we obtain

$$
\begin{aligned}
d(A-2 s A, B-2 s B) & \leq d(A, B)+2\|s A-s B\| \\
& \leq d(A, B)+2 \kappa_{n}\left\|\mathbb{B}^{e}\right\|^{2} d_{H}(A, B) \\
& \leq d(A, B)+2 \kappa_{n}\left\|\mathbb{B}^{e}\right\|^{2} d(A, B) .
\end{aligned}
$$

Let us denote $A(u)=\left\{a \in A \mid\langle a, u\rangle=\sup _{x \in A}\langle x, u\rangle\right\}$ for nonempty compact convex set $A$ and vector $u \in \mathbb{R}^{n}$. Let us notice that $m A+m B=m(A+B)$ is an extreme point of $A+B$ and a limit of some sequence $(A+B)\left(u_{n}\right)$ of exposed points. It is easy to see that $A\left(u_{n}\right)$ tends to $m A$ and $B\left(u_{n}\right)$ tends to $m B$. Hence

$$
\|m A-m B\| \leq \sup _{u \neq 0} d_{H}(A(u), B(u))=d_{D}(A, B) .
$$

For the last equality see [17] and [12]. Then

$$
d(A-2 m A, B-2 m B) \leq d(A, B)+2\|m A-m B\| \leq 3 d(A, B)
$$

for $d \in\left\{d_{D}, d_{B P}\right\}$.

In order to show discontinuity of $T_{M}$ with respect to Hausdorff metric let us consider $X=\mathbb{R}^{2}$, a segment $A_{0}$ with endpoints $(0,0)$ and $(0,1)$, and a sequence of segments $A_{n}$ with endpoints $(0,0)$ and $\left(-\frac{1}{n}, 1\right)$. Notice that $m A_{0}=(0,1)$ and $m A_{n}=$ $(0,0)$. In the same time $d_{H}\left(A_{n}, A_{0}\right)$ tends to 0 and $d_{H}\left(A_{n}-2 m A_{n}, A_{0}-2 m A_{0}\right)$ tends to $2\left\|m A_{n}-m A_{0}\right\|=2\|(0,-1)\|>0$.

Notice that if $A \subset B+\varepsilon \mathbb{B}$ then $R(A) \subset R(B)+\varepsilon R(\mathbb{B}) \subset R(B)+\varepsilon\|R(\mathbb{B})\| \mathbb{B}$. Hence $d_{H}\left(T_{R} A, T_{R} B\right) \leq\|R(\mathbb{B})\| d_{H}(A, B)$. The cases of $d_{D}$ and $d_{B P}$ are analogous.

It is easy to observe that constants in the table for $T_{0}, T_{M}$ and $T_{R}$ are the best possible. The authors believe that the problem of the best constants for $T_{S}$ is not trivial. Notice that we obtained decompositions with four different families of $T$ symmetric sets: $\{A \mid A=-A\},\{A \mid s A=0\},\{A \mid m A=0\},\{A \mid A=-A, R(A)=A\}$.

Theorem 3.5 A MRH space $\widetilde{X}$ is a topological direct sum of its symmetric and asymmetric subspaces for all pairs $(T, d) \in\left\{T_{0}, T_{S}, T_{M}, T_{R}\right\} \times\left\{d_{H}, d_{D}, d_{B P}\right\}$ of cone symmetry $T$ and metric $d$ with an exception of $\left(T_{M}, d_{H}\right)$.

Theorem 3.5 follows from Theorem 2.19 and Proposition 3.4. 
Theorem 3.5 suggests that decomposition of a MRH space into direct sum is possible for a variety of different cone symmetries $T$. Authors do not know how large is the family of all symmetries of $\mathcal{B}(X)$ that are uniformly continuous.

It is possible to interpret actions of symmetry $T_{0}$ on sublinear functions as mapping $f \mapsto g$, where $g(x)=f(-x)$. Then every difference $f$ of sublinear functions is decomposed into an even function $\frac{f(x)+f(-x)}{2}$ and an odd function $\frac{f(x)-f(-x)}{2}$. In the case of $T_{R}$ actions of symmetry can be interpreted as mapping $f \mapsto g$, where $g(x)=f(R(x))$.

\section{Application of Decomposition: Continuous Selection}

Let $\|\cdot\|$ be a norm in $\widetilde{X}$ and $d$ be a metric in $\mathcal{B}(X)$ such that $\|\widetilde{x}\|=d(A, B)$ for $\tilde{x}=[A, B], A, B \in \mathcal{B}(X)$. It would be very useful to be able to choose pairs of convex sets being representatives of elements $\widetilde{x}$ of the MRH space $\widetilde{X}$ in a continuous way. We can pose the following question: does there exist a function $\widetilde{X} \ni \widetilde{x} \longmapsto \Sigma(\widetilde{x})=$ $\left(\Sigma_{1}(\widetilde{x}), \Sigma_{2}(\widetilde{x})\right) \in \mathcal{B}^{2}(X)$ such that $\Sigma(\widetilde{x}) \in \widetilde{x}$ and $\Sigma_{1}, \Sigma_{2}$ are continuous with respect to the norm $\|\cdot\|$ and the metric $d$.

A quotient class $\widetilde{x} \in \widetilde{X}$ is partially ordered by inclusion, i.e. for $(A, B),(C, D)$ $\in \tilde{x}$ we have $(A, B) \prec(C, D)$ if and only if $A \subset C$ (and $B \subset D)$. If $X$ is a reflexive space then an inclusion minimal pair always exists [8]. If $X$ is two dimensional then a minimal pair is unique up to translation [7,24]. Therefore, some choice of minimal pair belonging to $\tilde{x}$ is a natural candidate for a function $\Sigma$.

The following example shows that the answer can be negative even for 2dimensional space $X$.

Example 4.1 Let $X=\mathbb{R}^{2}, A_{n}$ be a Euclidean disc with radius equal to $n, B_{n}$ be a regular $n^{2}$-gon inscribed in the disc $A_{n}, \widetilde{x}_{n}=\left[A_{n}, B_{n}\right]$. Notice that $\left\|\widetilde{x}_{n}\right\|_{D}$ tends to zero, hence $\widetilde{x}_{n}$ tends to $\tilde{0}$. Assume that $\Sigma(\widetilde{x}) \in \widetilde{x}$. Since all pairs $\left(A_{n}, B_{n}\right)$ are minimal then by existence and uniqueness of minimal pairs (see: [7, 15, 24]), the set $\Sigma_{1}\left(\widetilde{x}_{n}\right)$ contains some disc with radius $n$. Therefore, $\Sigma_{1}\left(\widetilde{x}_{n}\right)$ tends to no $\Sigma_{1}(\tilde{0})$ with respect to Hausdorff metric. Hence for no selection $\Sigma$ the functions $\Sigma_{1}, \Sigma_{2}$ are continuous with respect to norms weaker than $\|\cdot\|_{D}$ and metrics stronger than $d_{H}$.

Next example shows, that even with norm $\|\cdot\|_{B P}$ finding continuous function $\Sigma$ is not easy.

Example 4.2 Let $A_{n}$ be a triangle with vertices $(-1,0),(0,1)$ and $\left(0, \frac{1}{n}\right), B_{n}$ be a triangle with vertices $(1,0),(0,1)$ and $\left(0,-\frac{1}{n}\right), A$ be a triangle with vertices $(-1,0),(0,1)$ and $(0,0)$ and $B$ be a triangle with vertices $(1,0),(0,1)$ and $(0,0)$. Let $\widetilde{x}_{n}=\left[A_{n}, B_{n}\right]$ and $\widetilde{x}=[A, B]$. Since $\left(A_{n}+B, B_{n}+A\right) \sim\left(\{(0,0)\},\{0\} \times\left[-\frac{2}{n}, 0\right]\right)$, we have $\left\|\widetilde{x}_{n}-\tilde{x}\right\|_{B P}=\left\|\left[A_{n}+B, B_{n}+A\right]\right\|_{B P}=\frac{2}{n}$. It means that $\tilde{x}_{n}$ tends to $\tilde{x}$ with respect to Bartels-Pallaschke norm. If $\Sigma: \widetilde{\mathbb{R}^{2}} \longrightarrow \mathcal{B}^{2}\left(\mathbb{R}^{2}\right)$ such that $\Sigma(\widetilde{y}) \in \tilde{y}$ and $\left(\Sigma_{1}(\widetilde{y}), \Sigma_{2}(\widetilde{y})\right)$ is a minimal pair for all $\tilde{y}$ then by uniqueness of minimal pair $\Sigma_{1}\left(\widetilde{x}_{n}\right)=$ $A_{n}+u_{n}$ and $\Sigma_{1}(\widetilde{x})=A+u$ for some $u_{n}, u \in \mathbb{R}^{2}$. Then $d_{D}\left(\Sigma_{1}\left(\widetilde{x}_{n}\right), \Sigma_{1}(\widetilde{x})\right)=$ $d_{D}\left(A_{n}+u_{n}, A+u\right) \geq d_{H}\left(A_{n}(0,-1)+u_{n}, A(0,-1)+u\right) \geq \frac{1}{2}$ because $A_{n}(0,-1)$ is a singleton and $A(0,-1)$ is a segment of the length 1 . Therefore, $\Sigma_{1}$ is not continuous with respect to metric $d_{D}$ nor $d_{B P}$. 
However, our decomposition of an MRH-space over $\mathbb{R}^{2}$ combined with choosing minimal pairs enables us to prove an existence of continuous selection $\Sigma$ with respect to Bartels-Pallaschke norm and Bartels-Pallaschke metric.

Remark 4.3 We know that for any symmetric sets $A$ and $B$ a minimal pair $(C, D) \in$ $[A, B]$ exists and is unique up to translation. Since the pair $(-C,-D)$ is equivalent and also minimal, both $C$ and $D$ are centrally symmetric with the same center of symmetry. Therefore, the pair $(C+x, D+x) \in[A, B]$ is minimal and symmetric for some $x \in \mathbb{R}^{2}$.

In view of our remark we can formulate the following lemma.

Lemma 4.4 Let $A, B, C, D, \in \mathcal{B}\left(\mathbb{R}^{2}\right)$ be symmetric sets and $(A, B),(C, D)$ be minimal pairs. If $\left(G_{1}, G_{2}\right) \in[A, C],\left(H_{1}, H_{2}\right) \in[B, D]$ and $(E, F) \in[A+D, B+C]$ are minimal pairs and $G_{1}, G_{2}, H_{1}, H_{2}, E, F$ are symmetric sets then $E=G_{1}+H_{2}, F=$ $G_{2}+H_{1}$.

Proof Let $f_{A}:[0,2 \pi] \longrightarrow \mathbb{R}_{+}$be an arc length function of the boundary of $A$, that is

$$
f_{A}(t)=\sup _{P: 0=t_{0}<\ldots<t_{n}=t} \sum_{i=1}^{n}\left\|h_{A}\left(\cos t_{i}, \sin t_{i}\right)-h_{A}\left(\cos t_{i-1}, \sin t_{i-1}\right)\right\|,
$$

where $h_{A}(t)$ is the center of singleton or segment $A\left(\cos t_{i}, \sin t_{i}\right)$. Since $A$ and $B$ are symmetric, $f(\pi+t)=f(\pi)+f(t), x \in[0, \pi]$ for $f=f_{A}, f_{B}$ and $\inf \left(f_{A}, f_{B}\right)$. Let us remember that $g=\inf \left(f_{A}, f_{B}\right)$ is the greatest nondecreasing function such that $g(0)=0$ and functions $f_{A}-g, f_{B}-g$ are nondecreasing. Then minimality of the pair $(A, B)$ and Lemma 5.1 in [7] imply that $\inf \left(f_{A}, f_{B}\right)=0$. Also $\inf \left(f_{C}, f_{D}\right)=$ 0. Notice that $\inf \left(f_{A+D}, f_{B+C}\right)=\inf \left(f_{A}+f_{D}, f_{B}+f_{C}\right)=\inf \left(f_{A}, f_{C}\right)+\inf \left(f_{D}, f_{B}\right)$. By Theorem 3.3 in [9] we have $f_{E}=f_{A+D}-\inf \left(f_{A+D}, f_{B+C}\right)=f_{A}-\inf \left(f_{A}, f_{C}\right)+$ $f_{D}-\inf \left(f_{D}, f_{B}\right)=f_{G_{1}}+f_{H_{2}}$. By Proposition 4.5 in [7] we get $E=G_{1}+H_{2}+x$ for some $x \in \mathbb{R}^{2}$. Since the considered sets are symmetric, $x=0$. In a similar way we prove that $F=G_{2}+H_{1}$.

Lemma 4.5 Let $A, B \in \mathcal{B}\left(\mathbb{R}^{2}\right)$ and $(A,-A),(B,-B)$ be minimal pairs. If $\left(G_{1}, G_{2}\right) \in$ $[A, B]$ and $(E,-E) \in[A-B, B-A]$ are minimal pairs then $E+I=G_{1}-G_{2}$, where $I$ is a segment such that $I=-I$ and some translates of $I$ are contained respectively in the boundaries of $G_{1}$ and $G_{2}$.

Proof We have $f_{A}(\pi)+f_{-A}(t)=f_{A}(\pi+t)$ and $f_{-A}(\pi)+f_{A}(t)=f_{-A}(\pi+t)$ for $t \in[0, \pi]$. Then $\inf \left(f_{A}, f_{-A}\right)(\pi)+\inf \left(f_{A}, f_{-A}\right)(t)=\inf \left(f_{A}, f_{-A}\right)(\pi+t)$ for $t \in$ $[0, \pi]$. Hence by Lemma 5.1 in [7] we get $\inf \left(f_{A}, f_{-A}\right)=0$, and $\inf \left(f_{B}, f_{-B}\right)=0$.

Notice that

$$
\inf \left(f_{A-B}, f_{B-A}\right)=\inf \left(f_{A}+f_{-B}, f_{B}+f_{-A}\right)=\inf \left(f_{A}, f_{B}\right)+\inf \left(f_{-A}, f_{-B}\right) .
$$

Also inf $\left(f_{A}, f_{B}\right)(\pi)+\inf \left(f_{-A}, f_{-B}\right)(t)=\inf \left(f_{A}, f_{B}\right)(\pi+t)$ and $\inf \left(f_{-A}, f_{-B}\right)(\pi)+$ $\inf \left(f_{A}, f_{B}\right)(t)=\inf \left(f_{-A}, f_{-B}\right)(\pi+t)$ for $t \in[0, \pi]$. Both functions $\inf \left(f_{A}, f_{B}\right)$ and $\inf \left(f_{-A}, f_{-B}\right)$ are equal to 0 or take exactly three values and have exactly one point 
of discontinuity in $[0,2 \pi)$. Then $\inf \left(f_{A-B}, f_{B-A}\right)=$ has two points of discontinuity in $[0,2 \pi)$ which is impossible.

By Theorem 3.3 in [9] we obtain $f_{G_{1}}=f_{A}-\inf \left(f_{A}, f_{B}\right)+g$ and $f_{G_{2}}=f_{B}-$ $\inf \left(f_{A}, f_{B}\right)+g$, where $g$ is a nondecreasing function taking not more than three values. Then $f_{-G_{1}}=f_{-A}-\inf \left(f_{-A}, f_{-B}\right)+g^{\prime}$ and $f_{-G_{2}}=f_{-B}-\inf \left(f_{-A}, f_{-B}\right)+g^{\prime}$, where $g=g^{\prime}=0$ or the distance between points of discontinuity of $g$ and $g^{\prime}$ is equal to $\pi$. In that case $g+g^{\prime}=f_{I}$ for some symmetric segment $I$. Then translates of $I$ are contained in the boundaries of $G_{1}$ and $G_{2}$ on the same side.

Hence $f_{G_{1}}+f_{-G_{2}}=f_{A}-\inf \left(f_{A}, f_{B}\right)+g+f_{-B}-\inf \left(f_{-A}, f_{-B}\right)+g^{\prime}=f_{A-B}-$ $\inf \left(f_{A-B}, f_{B-A}\right)+f_{I}=f_{E}+f_{I}$. By Proposition 4.5 in [7] we have $E+I=G_{1}-$ $G_{2}+x$ for some $x \in \mathbb{R}^{2}$. Since $I$ is symmetric and $\left(G_{1}-G_{2}, G_{2}-G_{1}\right) \in[A, B]+$ $[-B,-A]=[A-B, B-A]=[E,-E]$, the vector $x$ is equal to 0 .

Now we can prove the main theorem of this section.

Theorem 4.6 There exists a function $\Sigma: \widetilde{\mathbb{R}^{2}} \longrightarrow \mathcal{B}^{2}\left(\mathbb{R}^{2}\right)$ such that $\Sigma(\widetilde{x})=$ $\left(\Sigma_{1}(\tilde{x}), \Sigma_{2}(\tilde{x})\right) \in \tilde{x}$ and $\Sigma_{1}, \Sigma_{2}$ are continuous with respect to the norm $\|\cdot\|_{B P}$ and the metric $d_{B P}$.

Proof Let $\Phi: \widetilde{\mathbb{R}^{2}}{ }_{s} \longrightarrow \mathcal{B}^{2}\left(\mathbb{R}^{2}\right)$ be such a function that $\left(\Phi_{1}(\widetilde{x}), \Phi_{2}(\widetilde{x})\right)$ is a minimal pair and $\Phi_{1}(\widetilde{x})=-\Phi_{1}(\widetilde{x}), \Phi_{2}(\widetilde{x})=-\Phi_{2}(\widetilde{x})$. Let $\Psi: \widetilde{\mathbb{R}^{2}}{ }_{a} \longrightarrow \mathcal{B}^{2}\left(\mathbb{R}^{2}\right)$ be such a function that $\left(\Psi_{1}(\widetilde{x}), \Psi_{2}(\widetilde{x})\right)$ is a minimal pair and $\Psi_{1}(\widetilde{x})=-\Psi_{2}(\widetilde{x})$. By existence and uniqueness-up-to-translation of minimal pairs such functions exist. We define $\Sigma$ : $\widetilde{\mathbb{R}^{2}} \longrightarrow \mathcal{B}^{2}\left(\mathbb{R}^{2}\right)$ by $\Sigma_{1}(\widetilde{x})=\Phi_{1}\left(\widetilde{x}_{s}\right)+\Psi_{1}\left(\widetilde{x}_{a}\right), \Sigma_{2}(\widetilde{x})=\Phi_{2}\left(\widetilde{x}_{s}\right)-\Psi_{1}\left(\widetilde{x}_{a}\right)$.

Let $\widetilde{x}, \widetilde{y} \in \widetilde{\mathbb{R}^{2}},(E, F) \in\left[\Phi_{1}\left(\widetilde{x}_{s}\right)+\Phi_{2}\left(\widetilde{y}_{s}\right), \Phi_{1}\left(\widetilde{y}_{s}\right)+\Phi_{2}\left(\widetilde{x}_{s}\right)\right]$ be a minimal pair with $E=-E, F=-F,(\bar{E},-\bar{E}) \in\left[\Psi_{1}\left(\widetilde{x}_{a}\right)-\Psi_{1}\left(\widetilde{y}_{a}\right), \Psi_{1}\left(\widetilde{y}_{a}\right)-\Psi_{1}\left(\widetilde{x}_{a}\right)\right]$ be a minimal pair. Moreover, let $\left(G_{1}, G_{2}\right) \in\left[\Phi_{1}\left(\widetilde{x}_{s}\right), \Phi_{1}\left(\widetilde{y}_{s}\right)\right],\left(H_{1}, H_{2}\right) \in\left[\Phi_{2}\left(\widetilde{x}_{s}\right), \Phi_{2}\left(\widetilde{y}_{s}\right)\right],\left(\bar{G}_{1}, \bar{G}_{2}\right) \in$ $\left[\Psi_{1}\left(\widetilde{x}_{a}\right), \Psi_{1}\left(\widetilde{y}_{a}\right)\right]$ be minimal pairs such that $d_{B P}\left(\Phi_{1}\left(\widetilde{x}_{s}\right), \Phi_{1}\left(\widetilde{y}_{s}\right)\right)=\left\|G_{1}\right\|+\left\|G_{2}\right\|$, $d_{B P}\left(\Phi_{2}\left(\widetilde{x}_{s}\right), \Phi_{2}\left(\widetilde{y}_{s}\right)\right)=\left\|H_{1}\right\|+\left\|H_{2}\right\|$, $d_{B P}\left(\Psi_{1}\left(\widetilde{x}_{a}\right), \Psi_{1}\left(\widetilde{y}_{a}\right)\right)=\left\|\bar{G}_{1}\right\|+\left\|\bar{G}_{2}\right\|$.

By Lemma 4.4 we have

$$
\begin{aligned}
d_{B P}\left(\Phi_{1}\left(\widetilde{x}_{s}\right), \Phi_{1}\left(\widetilde{y}_{s}\right)\right) & =\left\|G_{1}\right\|+\left\|G_{2}\right\| \\
& \leq\left\|G_{1}+H_{2}\right\|+\left\|H_{1}+G_{2}\right\|=\|E\|+\|F\| \\
& =\left\|\left[\Phi_{1}\left(\widetilde{x}_{s}\right)+\Phi_{2}\left(\widetilde{y}_{s}\right), \Phi_{1}\left(\widetilde{y}_{s}\right)+\Phi_{2}\left(\widetilde{x}_{s}\right)\right]\right\|_{B P} \\
& =\left\|\left[\Phi_{1}\left(\widetilde{x}_{s}\right), \Phi_{2}\left(\widetilde{x}_{s}\right)\right]-\left[\Phi_{1}\left(\widetilde{y}_{s}\right), \Phi_{2}\left(\widetilde{y}_{s}\right)\right]\right\|_{B P} \\
& =\left\|\widetilde{x}_{s}-\widetilde{y}_{s}\right\|_{B P} .
\end{aligned}
$$

In a similar way, $d_{B P}\left(\Phi_{2}\left(\widetilde{x}_{s}\right), \Phi_{2}\left(\widetilde{y}_{s}\right)\right) \leq\left\|\widetilde{x}_{s}-\widetilde{y}_{s}\right\|_{B P}$.

Since for all $x$ we have $\left\|\bar{G}_{1}+x\right\|+\left\|\bar{G}_{2}+x\right\| \geq\left\|\bar{G}_{1}\right\|+\left\|\bar{G}_{2}\right\|$, there exist $u \in$ $\bar{G}_{1}, v \in \bar{G}_{2}$ such that 0 belongs to the segment with endpoints $u$ and $v$. By Lemma 4.5, and since $E$ contains some translate of $I$, we have

$$
\begin{aligned}
d_{B P}\left(\Psi_{1}\left(\widetilde{x}_{a}\right), \Psi_{1}\left(\widetilde{y}_{a}\right)\right) & =\left\|\bar{G}_{1}\right\|+\left\|\bar{G}_{2}\right\| \\
& \leq\left\|\bar{G}_{1}-u\right\|+\|u\|+\left\|\bar{G}_{2}-v\right\|+\|v\| \\
& \leq \operatorname{diam}\left(\bar{G}_{1}\right)+\operatorname{diam}\left(\bar{G}_{2}\right)+\|u-v\|
\end{aligned}
$$




$$
\begin{aligned}
& \leq 3 \operatorname{diam}\left(\bar{G}_{1}-\bar{G}_{2}\right) \leq 3 \operatorname{diam}(\bar{E}+I) \\
& \leq 6 \operatorname{diam}(\bar{E}) \leq 12\|\bar{E}\| \leq 6(\|\bar{E}\|+\|-\bar{E}\|) \\
& =6\left\|\left[\Psi_{1}\left(\widetilde{x}_{a}\right)-\Psi_{1}\left(\widetilde{y}_{a}\right), \Psi_{1}\left(\widetilde{y}_{a}\right)-\Psi_{1}\left(\widetilde{x}_{a}\right)\right]\right\|_{B P} \\
& =6\left\|\left[\Psi_{1}\left(\widetilde{x}_{a}\right),-\Psi_{1}\left(\widetilde{x}_{a}\right)\right]-\left[\Psi_{1}\left(\widetilde{y}_{a}\right), \Psi_{1}\left(\widetilde{y}_{a}\right)\right]\right\|_{B P} \\
& =6\left\|\widetilde{x}_{a}-\widetilde{y}_{a}\right\|_{B P .} .
\end{aligned}
$$

In a similar way, $d_{B P}\left(-\Psi_{1}\left(\widetilde{x}_{a}\right),-\Psi_{1}\left(\widetilde{y}_{a}\right)\right) \leq 6\left\|\widetilde{x}_{a}-\widetilde{y}_{a}\right\|_{B P}$.

Then

$$
\begin{aligned}
d_{B P}\left(\Sigma_{1}(\widetilde{x}), \Sigma_{1}(\widetilde{y})\right) & =\left\|\left[\Sigma_{1}(\widetilde{x}), \Sigma_{1}(\widetilde{y})\right]\right\|_{B P} \\
& =\left\|\left[\Phi_{1}\left(\widetilde{x}_{s}\right)+\Psi_{1}\left(\widetilde{x}_{a}\right), \Phi_{1}\left(\widetilde{y}_{s}\right)+\Psi_{1}\left(\widetilde{y}_{a}\right)\right]\right\|_{B P} \\
& \leq\left\|\left[\Phi_{1}\left(\widetilde{x}_{s}\right), \Phi_{1}\left(\widetilde{y}_{s}\right)\right]\right\|_{B P}+\left\|\left[\Psi_{1}\left(\widetilde{x}_{a}\right), \Psi_{1}\left(\widetilde{y}_{a}\right)\right]\right\|_{B P} \\
& =d_{B P}\left(\Phi_{1}\left(\widetilde{x}_{s}\right), \Phi_{1}\left(\widetilde{y}_{s}\right)\right)+d_{B P}\left(\Psi_{1}\left(\widetilde{x}_{a}\right), \Psi_{1}\left(\widetilde{y}_{a}\right)\right) \\
& \leq\left\|\widetilde{x}_{s}-\widetilde{y}_{s}\right\|_{B P}+6\left\|\widetilde{x}_{a}-\widetilde{y}_{a}\right\|_{B P} \leq 7 \| \widetilde{x}-\widetilde{y}_{B P} .
\end{aligned}
$$

In a similar way, $d_{B P}\left(\Sigma_{2}(\widetilde{x}), \Sigma_{2}(\widetilde{y})\right) \leq 7\|\widetilde{x}-\widetilde{y}\|_{B P}$.

The following table summarizes the answer to the question: does there exist a continuous selection $\Sigma$ from a normed MRH space $\widetilde{\mathbb{R}^{2}}$ to $\mathcal{B}^{2}\left(\mathbb{R}^{2}\right)$ with respect to norm $\|\cdot\|$ and metric $d$ ?

\begin{tabular}{|l|c|c|c|}
\hline & $d_{H}$ & $d_{D}$ & $d_{B P}$ \\
\hline$\|\cdot\|_{H}$ & no & no & no \\
\hline$\|\cdot\|_{D}$ & no & no & no \\
\hline$\|\cdot\|_{B P}$ & yes & yes & yes \\
\hline
\end{tabular}

We can apply different decomposition to prove the existence of a continuous selection $\Sigma$ from a normed MRH space $\widetilde{\mathbb{R}^{2}}$ to the family of minimal pairs in $\mathcal{B}\left(\mathbb{R}^{2}\right)^{2}$ with respect to norm $\|\cdot\|_{B P}$ and metric $d_{H}$.

Proposition 4.7 Let $\mathcal{A}$ be a family of non-decreasing functions $f$ defined on $[0,2 \pi]$ that

(i) $f(0)=0$

(ii) $\lim _{t \rightarrow a^{-}} f(t)=\lim _{t \rightarrow a^{+}} f(t)$ for all $a \in(0,2 \pi)$

(iii) $\lim _{t \rightarrow 0^{+}} f(t)+\lim _{t \rightarrow 2 \pi^{-}} f(t)=f(2 \pi)$.

Let $A, B \in \mathcal{B}\left(\mathbb{R}^{2}\right)$ and $f_{A}=f+g$ where $f, g \in \mathcal{A}$ and $g$ takes exactly three values. If $f \prec f_{B}$ then $A+x \subset B$ for some $x \in \mathbb{R}^{2}$.

We omit the proof which uses techniques applied in [7].

Let us consider the symmetry $T=T_{S}$. For $\widetilde{x} \in \widetilde{\mathbb{R}^{2}}{ }_{s}$ we define $\Phi(\widetilde{x})=(A, B)$ where the pair $(A, B) \in \tilde{x}$ is a minimal pair such that $s A=s B=0$. Such a pair exists and it is unique. The following lemma holds true. 
Lemma 4.8 Let $\Phi(\widetilde{x})=\left(\Phi_{1}(\widetilde{x}), \Phi_{2}(\widetilde{x})\right)$. Then the functions $\Phi_{1}, \Phi_{2}: \widetilde{\mathbb{R}^{2}}{ }_{s} \longrightarrow \mathcal{B}\left(\mathbb{R}^{2}\right)$ are continuous with respect to Bartels-Pallaschke norm $\|\cdot\|_{B P}$ and Hausdorff metric $d_{H}$.

Proof Let $\tilde{x}, \widetilde{y} \in \widetilde{\mathbb{R}^{2}}{ }_{s}, \quad \Phi(\widetilde{x})=(A, B), \Phi(\widetilde{y})=(C, D) \quad$ and $\quad\|\widetilde{x}-\widetilde{y}\|_{B P}=\varepsilon$. By definition of Bartels-Pallaschke norm there exist $(E, F) \in \tilde{x}-\tilde{y}$ such that $\|E\|+\|F\|=\varepsilon$. We have

$$
A+D+F=B+C+E .
$$

Then $f_{A}-\inf \left(f_{A}, f_{B}\right) \prec f_{C}+f_{E}$. By Proposition 4.7 we obtain

$$
A+u \subset C+E
$$

for some $u \in \mathbb{R}^{2}$. In a similar way

$$
C+v \subset A+F
$$

for some $v \in \mathbb{R}^{2}$. Then

$$
A+u+v \subset C+E+v \subset A+E+F .
$$

Hence $u+v \in E+F$ and $\|u+v\| \leq \varepsilon$. Then $A+u \in C+\varepsilon \mathbb{B}$ and

$$
C=C+u+v-(u+v) \subset A+F+u-(u+v) \subset A+u+2 \varepsilon \mathbb{B}
$$

where $\mathbb{B}$ is the Euclidean unit disc in $\mathbb{R}^{2}$. Therefore, $d_{H}(A+u, C) \leq 2 \varepsilon$. On the other hand

$$
d_{H}(A+u, A)=\|u\|=\|s(A+u)-s C\| \leq \frac{4}{\pi} d_{H}(A+u, C) \leq \frac{8}{\pi} \varepsilon .
$$

Therefore,

$$
d_{H}\left(\Phi_{1}(\widetilde{x}), \Phi_{1}(\widetilde{y})\right)=d_{H}(A, C) \leq 5 \varepsilon=5\|\widetilde{x}-\widetilde{y}\|_{B P} .
$$

In a similar way we can prove that $d_{H}\left(\Phi_{2}(\widetilde{x}), \Phi_{2}(\tilde{y})\right) \leq 5\|\tilde{x}-\tilde{y}\|_{B P}$.

Theorem 4.9 There exists a function $\Sigma: \widetilde{\mathbb{R}^{2}} \longrightarrow \mathcal{B}^{2}\left(\mathbb{R}^{2}\right)$ with $\Sigma(\widetilde{x})=\left(\Sigma_{1}(\widetilde{x}), \Sigma_{2}(\widetilde{x})\right) \in$ $\widetilde{x}$ such that all pairs $\left(\Sigma_{1}(\widetilde{x}), \Sigma_{2}(\widetilde{x})\right)$ are minimal and $\Sigma_{1}, \Sigma_{2}$ are continuous with respect to the norm $\|\cdot\|_{B P}$ and the metric $d_{H}$.

Proof Let us put $\Sigma(\widetilde{x})=\Phi\left(\widetilde{x}_{s}\right)$, where $\Phi$ is a selection from Lemma 4.8. Our theorem follows from Theorem 2.19, Proposition 3.4 and Lemma 4.8.

The following table summarizes the answer to the question: does there exist a continuous selection $\Sigma$ from a normed MRH space $\widetilde{\mathbb{R}^{2}}$ to the family of minimal pairs in $\mathcal{B}\left(\mathbb{R}^{2}\right)^{2}$ with respect to norm $\|\cdot\|$ and metric $d$ ?

\begin{tabular}{|l|c|c|c|}
\hline & $d_{H}$ & $d_{D}$ & $d_{B P}$ \\
\hline$\|\cdot\|_{H}$ & no & no & no \\
\hline$\|\cdot\|_{D}$ & no & no & no \\
\hline$\|\cdot\|_{B P}$ & yes & no & no \\
\hline
\end{tabular}


Unfortunately in $\mathbb{R}^{3}$ in general there is no uniqueness-up-to-translation of minimal pairs of compact convex sets [7]. Theorems similar to Theorems 4.6 and 4.9 would require a different approach.

Acknowledgement The authors thank the anonymous referee for his comments, which helped to improve the paper.

Open Access This article is distributed under the terms of the Creative Commons Attribution License which permits any use, distribution, and reproduction in any medium, provided the original author(s) and the source are credited.

\section{References}

1. Demyanov, V.F., Rubinov, A.M.: Quasidifferential Calculus. Optimization Software, New York (1986)

2. Demyanov, V.F., Rubinov, A.M.: Quasidifferentiability and related topics. In: Nonconvex Optim. Appl. Kluwer, Dordrecht (2001)

3. Diamond, P., Kloeden, P., Rubinov, A., Vladimirov, A.: Comparative properties of three metrics in the space of compact convex sets. Set-Valued Anal. 5, 267-289 (1997)

4. Drewnowski, L.: Embeddings of topological semigroups in topological groups and semigroupvalued measures. Demonstr. Math. 14(2), 355-360 (1981)

5. Ewald, G., Shephard, G.C.: Normed vector spaces consisting of classes of convex sets. Math. Zeitschr. 91, 1-19 (1966)

6. Gorokhovik, V.V., Zabreiko, P.P.: Fréchet differentiability of multifunctions. Optimization 54, 391-409 (2005)

7. Grzybowski, J.: Minimal pairs of compact compact convex sets. Arch. Math. 63, 173-181 (1994)

8. Grzybowski, J., Urbański, R.: Minimal pairs of bounded closed convex sets. Stud. Math. 126, 95-99 (1997)

9. Grzybowski, J., Pallaschke, D., Urbański, R.: Minimal pairs of bounded closed convex sets as minimal representations of elements of the Minkowski-Rådström-Hörmander spaces. Banach Center Publ. 84(1), 31-55 (2009)

10. Grzybowski, J., Pallashke, D., Przybycień, H., Urbański, R.: Commutative semigroups with cancellation law: a representation theorem. Semigroup Forum 83(3), 447-456 (2011)

11. Hörmander, L.: Sur la fonction d'appui des ensembles convexes dans un espace localement convexe. Ark. Math. 3,181-186 (1954)

12. Leśniewski, A., Rzeżuchowski, T.: Demyanov's metric for convex, bounded sets and existence of Lipschitzian selectors. J. Convex Anal. 18(3), 737-747 (2011)

13. Markov, S.: On quasilinear spaces of convex bodies and intervals. J. Comput. Appl. Math. 162, 93-112 (2004)

14. Mordukhovich, B.S.: Variational Analysis and Generalized Differentiation. Springer, Berlin (2006)

15. Pallaschke, D., Scholtes, S., Urbański, R.: On minimal pairs of convex compact sets. Bull. Pol. Acad. Sci., Math. 39, 1-5 (1991)

16. Pallaschke, D., Urbański, R.: Pairs of compact convex sets: fractional arithmetic with convex sets. In: Math. Appl., vol. 548. Kluwer, Dordrecht (2002)

17. Pliś, A.: Uniqueness of optimal trajectories for non-linear control problems. Ann. Pol. Math. 29, 397-401 (1975)

18. Przesławski, K.: Linear and Lipschitz continuous selectors for the family of convex sets in Euclidean vector spaces. Bull. Pol. Acad. Sci., Math. 33, 31-33 (1985)

19. Rabinovich, M.G.: Some classes of spaces of convex sets and their extensions. Sib. Math. J. 8, 1064-1070 (1967)

20. Rådström, H.: An embedding theorem for spaces of convex sets. Proc. Am. Math. Soc. 3, 165-169 (1952)

21. Ratschek, H., Schröder, G.: Representation of semigroups as systems of compact convex sets. Proc. Am. Math. Soc. 65(1), 24-28 (1977)

22. Rockafellar, R.T., Wets, R.J.-B.: Variational Analysis. Springer, Berlin (1998) 
23. Schneider, S.: Convex bodies: the Brunn-Minkowski theory. In: Encyclopedia of Mathematics and its Applications, vol. 44. Cambridge University Press, Cambridge (1993)

24. Scholtes, S.: Minimal pairs of convex bodies in two dimensions. Mathematika 39, 267-273 (1992)

25. Urbański, R.: A generalization of the Minkowski-Rådström-Hörmander theorem. Bull. Acad. Pol. Sci., Sér. Sci. Math. Astron. Phys. 24, 709-715 (1976) 